\section{Antibiotic resistance must be monitored, US Senate is told}

\section{Paul Smaglik, Washington}

The United States needs a better monitoring system to combat the threat posed by the rising number of bacteria resistant to existing antibiotics, federal officials told a Senate hearing last week.

Apart from drug-resistant tuberculosis, which is tracked in all 50 states, monitoring of antibiotic-resistant microbes remains relatively fragmented.

According to the limited data available, drug resistance can vary widely, even within states. In Maryland, for example, $15 \%$ of the strains of Streptococcus pneumoniae, the bacterium that causes pneumonia and meningitis, are resistant to penicillin. In five Tennessee counties, resistance is seen in $38 \%$ of the strains.

But this kind of reporting is not the norm, Jeffrey Koplan, director of the Centers for Disease Control and Prevention (CDC), told a hearing of the Senate Appropriations Subcommittee on Labor, Health and Human Services, and Education. Better monitoring, he said, as well as more research into how microbes develop resistance, needs to be stressed in the next federal budget.

New forms of drug resistance continue to develop, Koplan explained. For example, a CDC report last week identified two strains of Neisseria gonorrhoeae, the bacterium that causes gonorrhoea, that have recently become resistant to some of the drugs used to treat the condition.

Jane Henney, US Food and Drug Administration (FDA) commissioner, agreed that surveillance is a key part of fighting drug-resistant microbes. Spotting resistant strains earlier allows doctors to prescribe different antibiotics, which is important, as using ineffective antibiotics promotes further resistance.

Doctors need to be more aware of the dangers of prescribing antibiotics unnecessarily, and patients should be educated about their proper use, Henney said. She also noted the need for faster development of new vaccines and antibiotics.

It remains unclear which strategies will be given highest priority in the US administration's next budget request. But the FDA, CDC, National Institutes of Health and seven other federal agencies formed a task force on drug resistance last summer. This has since released a series of recommendations, including the need for a better system to monitor drugresistant pathogens.

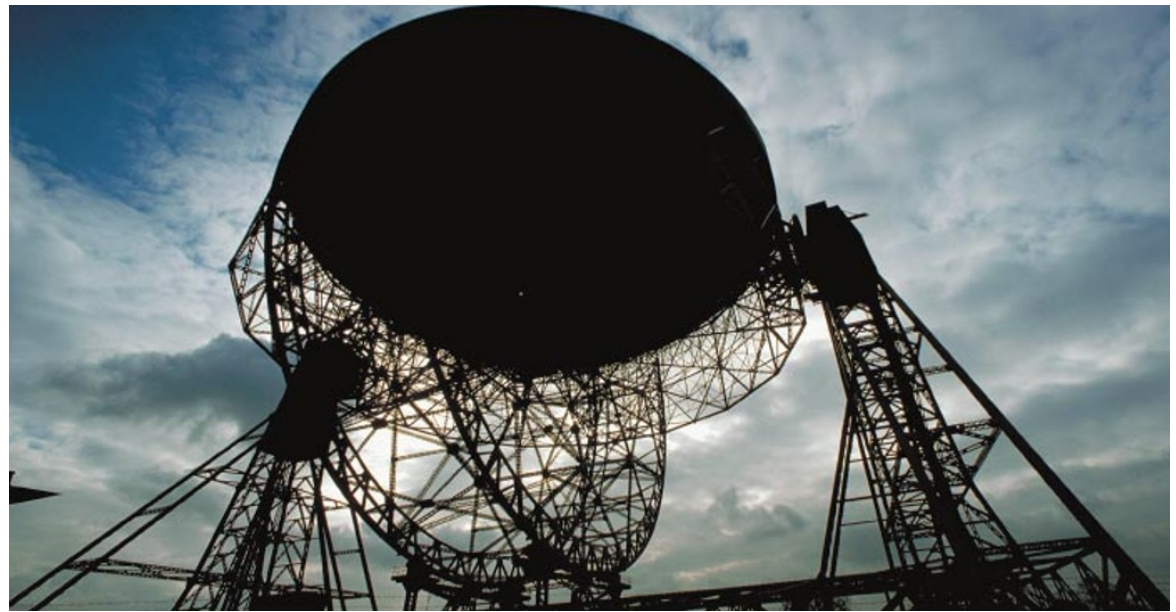

Team member: the Jodrell Bank radio telescope, one of 18 telescopes that form part of the network.

\title{
Report praises European radio telescope network
}

Alison Abbott

A European network of radio telescopes that allows small celestial objects to be imaged at very high resolution is a major scientific success, according to a review published last week. But the report also says the network needs secure, long-term funding if it is to provide optimal value to its growing user community.

The European Very-Long-Baseline Interferometry Network (EVN) was set up on an ad hoc basis in the 1980s by 14 astronomy institutes. The network's telescopes dedicate up to $30 \%$ of their observation time to simultaneous data-gathering, greatly increasing the resolution of observed images.

The resolving power of a telescope is limited by its physical diameter. Very-long-baseline interferometry is a technique that, by linking up distant telescopes, increases the diameter of their collecting area to the distance between them.

The EVN links 18 radio telescopes in this way, including three of the world's largest, which have diameters of 100 metres (at Effelsberg, near Bonn), 94 metres (Westerbork, the Netherlands) and 76 metres (Jodrell Bank, United Kingdom).

Last week's review was commissioned by the Netherlands national research council on behalf of the research councils involved in the EVN, and was carried out by the European Science Foundation. It was aided by an international panel chaired by Jens Erik Fenstad, a mathematician at the University of Oslo.

The report's praise for the network extends to astronomers working in wavelengths other than radio. One example is the EVN's detection of 50 supernovae in M82, a relatively close galaxy whose central regions

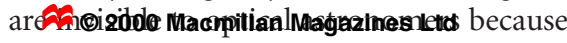
the galaxy is very 'dusty'.
"We can watch the gas that forms when a supernova remnant expands, allowing us to study the physics of star formation and star death," says Harvey Butcher, director of the Netherlands Foundation for Research in Astronomy at Dwingeloo.

Although initial funding for the EVN came from its participating research institutes, the network has since been designated a European Union Large Scale Facility. This allows the European Commission to support scientists' access to the facility. The network has also raised funding from various sources to develop data-processing technologies.

Last year, for example, the EVN brought online the world's largest 'correlator', which merges data the telescopes have collected on magnetic tape and converts them to images. The correlator is based at the Joint Institute for VLBI in Europe (JIVE) in Dwingeloo.

At present, however, half of JIVE's operating budget is paid for by a consortium of seven national research institutes through shortterm bilateral agreements which expire at different times. The rest comes from sources that have been gathered by EVN institutes.

Such a funding policy basis is "too frail" for a key facility such as JIVE, says the report. According to Phil Diamond, a director at the Jodrell Bank Observatory and a member of the report's panel of experts, national research councils must at least double their contribution to JIVE - currently around 700,000 euros (US\$615,000) — and operate through a single, multinational agreement.

Further sources of income would also be needed, he says, if EVN-JIVE is to develop its technologies and services.

Butcher believes that the report should help ENV-JIVE in its fight for a simple and stable source of funding.

http://www.esf.org/update/news/00/jive.htm 